\title{
Neue Dialoge
}

\section{Das ABC von Gilles Deleuze}

Antonia von Schöning/Hanns ZischleR

1988 gibt Gilles Deleuze seine hartnäckige Weigerung auf, im Fernsehen aufzutreten und lässt sich auf ein über siebenstündiges Filmgespräch mit seiner ehemaligen Studentin und Freundin Claire Parnet ein. Seine Bedingung dafür ist, dass das Material erst nach seinem Tod ausgestrahlt werden darf. Dass Deleuze einige Monate vor seinem krankheitsbedingten Freitod 1995 der Ausstrahlung in einzelnen Folgen dennoch zustimmt, ändert nichts an der ursprünglichen Versuchsanordnung dieses besonderen Filmdokuments, in dem Deleuze den Impulsen der alphabetischen Begriffsauswahl von Claire Parnet von A wie Animal bis $Z$ wie Zickzack folgt und ausführlich zu so heterogenen Themen wie »Kultur«, »Geschichte der Philosophie«, »Literatur« oder »Widerstand «spricht. Nachdem das von Pierre-André Boutang produzierte TV-Interview L'Abécédaire de Gilles Deleuze in Frankreich seit seiner Erscheinung 1988 auf Video und später auf DVD bekannt ist und großen Erfolg hat, ist 2009 eine längst überfällige deutsche Version erschienen. Die Übersetzung des vollständigen Dokuments von Valeska Bertoncini bewegt sich dabei faszinierend nah an der Vorlage des Originals. Hanns Zischler und Antonia von Schöning haben eine Voice-Over-Fassung gesprochen.

Hanns Zischler: Ich möchte nicht das Wort ergreifen, sondern fortsetzen, was wir schon einmal gemacht haben. Wir haben beide mit verteilten Rollen oder besser mit verteilten Stimmen die deutsche Übersetzung des Abécédaire gesprochen, und zwar entlang dem französischen Original, also synchron. 
Und jetzt frage ich dich als mein weibliches Gegenüber - in Vertretung von Claire Parnet - was geschieht, wenn man ein solches Gespräch, wie es zwischen Gilles Deleuze und Claire Parnet stattgefunden hat, fortlaufend in eine andere Sprache transponiert.

Antonia von Schöning: Genau diese Frage stellt sich, weil es in keiner Weise darum ging, das originale Gespräch nachzuahmen. Wir wiederholen vielmehr frei nach Deleuze und dem Schema der Wiederholung und Differenz eine Gesprächs- und Sprechsituation, aber verändern sie zugleich. Dadurch, dass wir andere sind und eine andere Sprache sprechen, ist es natürlich auch ein anderes Gespräch geworden. Zugleich geht es auch um die Frage, wie überhaupt über Philosophie gesprochen werden kann. Deleuze und Parnet stellen sie, und wir stellen sie noch einmal.

HZ: Ja, es ist ein anderes Gespräch geworden und gleichzeitig ist es natürlich gefasst und vorgegeben durch eine bereits vorhandene Aufzeichnung, das heißt durch diesen Film, der zu Deleuzes Lebzeiten eigentlich nicht gezeigt werden sollte.

Es gibt für mich bei dieser Art von begleitender beziehungsweise gleitender Übersetzung ein Problem: dass der Sprechakt von beiden - von Deleuze naturgemäß mehr als von Claire - durch die lebendige wörtliche Rede gekennzeichnet ist. Und sie ist durchsetzt von unzählig vielen Akzenten, die wiederum eine Bedeutung schaffen, einen bestimmten Sinn herstellen. Unser Sprechen dagegen ist ja keine lebendige Rede in diesem Sinn.

AS: Ja, für eine nachträgliche, deutsche Version stellt sich dieses Problem, das wir aber nicht zu vertuschen versuchen. Andererseits ist es ein spannendes Experiment, in diese philosophische Praxis außerhalb der Schrift mit einzusteigen, sie zu entfremden und ihr etwas hinzuzufügen. Durch die Verschiebung und Übersetzung, die wir erlebt haben, indem wir eine eigentlich gesprochene, lebendige Sprache übernehmen und sie mitlesen, passiert etwas Produktives mit dem ursprünglichen Sprechakt.

HZ: Es passiert das, was in der Synchronisation - ich nenne das jetzt mal so - immer schon kritisiert worden ist. Borges hat einmal gesagt, Synchronisation ist eine Amputation, ein Bauchreden sozusagen. Der Körper ist von seiner Stimme getrennt beziehungsweise die Stimme als der individu- 
elle Teil des Menschen vom Körper als dem sozialen Teil. Daher rührt auch dieses schreckliche Unbehagen für mich, durch »Synchronisation« (die ja eigentliche eine Entzweiung ist) betrogen, mit einem falschen Text hereingelegt zu werden. Kann man überhaupt diesem langen, ausführlichen Gespräch, so wie es stattgefunden hat und aufgezeichnet wurde, gerecht werden in einer gesprochenen Form?

AS: Man muss dazu sagen, dass das Gespräch - ob in französischer oder deutscher Sprache - bis heute nicht verschriftlicht werden darf. Auch dieses Verbot eines Medienwechsels macht das ABC zu einem sehr besonderen Dokument. Laß' uns doch einmal die Gesprächssituation zwischen Gilles Deleuze und Claire Parnet genauer betrachten. Sie entsteht ja unter der Voraussetzung der posthumen Veröffentlichung. Deleuze lässt sich darauf ein, weil er weiß, dass die Aufzeichnung erst nach seinem Tod veröffentlicht wird, eine Tatsache, die ihm, wie er sagt, die Freiheit gibt zu sprechen. Er bezeichnet sich als »Stück Papier«, als »Archiv« bereits zu Lebzeiten und als »reiner Geist«, wie er ironisch formuliert. Das ist also die Voraussetzung für dieses Gespräch und dann kommt das ganze Setting hinzu, das auch beinhaltet, dass man ihn über Stunden in unterschiedlich nahen Einstellungen sieht, Claire Parnet dagegen entweder in einem Spiegel hinter ihm oder angeschnitten im Blick über ihre Schulter. Hauptsächlich guckt man Claire Parnet über die Schulter. Wenn wir schließlich in die Dialogsituation mit einsteigen, kommt noch der Aspekt des körperlosen Sprechens hinzu, der aber durch die Klausel der posthumen Veröffentlichung auch in der Originalversion schon vorhanden ist.

HZ: Es hat etwas Unheimliches für mich. Ob ironisch, kokett oder leicht hingesagt, Deleuze betont es mehrere Male, dass er posthum oder von »après ma mort« spricht, das heißt: Ich spreche jetzt aus dem Jenseits. Ich spreche als Toter zu euch. Was ein sehr ungewöhnlicher Vorgang ist. Ungewöhnlich nicht, weil es diese Situation noch nie gegeben hätte, sondern ungewöhnlich ist, im Bewusstsein dessen zu sprechen. Die beiden wissen von vornherein, dass sie als Geisterstimmen zu uns sprechen. Das heißt, Deleuze formuliert in einem sehr, sehr langen Gespräch sein Testament, »the dying word « nicht angesichts, sondern in Antizipation seines Todes. Und jetzt kommen wir als Sprecher aus dem Off und transplantieren das Ganze mit Hilfe der Übersetzung und der Technik in eine andere Gegenwart. Im Grunde unterlaufen wir das Testament. Also ich weiß, wenn ich 
beim Film oder bei Aufnahmen für die Kamera spreche, wenn ich etwas mitteile, dass dies natürlich irgendwann auch noch zu sehen und zu hören sein wird, wenn ich nicht mehr da bin. Aber das ist in der Regel ohne Belang für die Aufnahme, auch für die Mitteilung selbst. Während das in diesem sehr besonderen Fall ein zentraler und essentieller Teil der Mitteilung selbst.

AS: Hier ist es die Bedingung ihrer Möglichkeit. Unter anderen Voraussetzungen hätte sich Deleuze auf eine Aufzeichnung dieses Gesprächs wohl nicht eingelassen. Und er sagt ja, es ist befreiend für ihn, für sein Sprechen. Aber du hast völlig Recht, die Wiederholung davon ist besonders gespenstisch, weil die Bedingung, also die Bedingung des eigenen Todes so explizit im Raum steht.

HZ: Ja, die steht im Raum und ist auch fortwährend zu spüren. Man weiß es, weil man es gleich zu Anfang gehört hat. Ich glaube, es kommt noch etwas hinzu: Es ist im Allgemeinen verpönt, so etwas zu sagen. Man kann nicht umhin, sich zu fragen, ob Deleuze den Gedanken an seinen Freitod mit einbezogen hat in diese Mitteilung »nach meinem Tod«. Nach konventionellen Vorstellungen wird über den eigenen Tod nicht gesprochen. Beim Schreiben ist das etwas anderes. Die Schrift ist das, was dem Autor von vornherein schon überleben wird. Die Schrift hat sozusagen das Jenseitige schon in sich, das Spätere, das, was vom Autor fortleben soll. Dieses Gespräch aber, dieser Dialog lebt geradezu von einer Frénésie, einer Raserei der Rede und von einer uneinholbaren Präsenz des gesprochenen Wortes - fast ist es, als wollten Deleuze und Parnet diese Emphase für die Aufzeichnung noch verstärken. Das fortlaufende und darunter laufende Bewusstsein, dass es für ein Danach gedacht ist, macht mir immer wieder zu schaffen.

Ich weiß nicht, was geschieht, wenn wir das im Deutschen nachsprechen. Ich habe den Eindruck, dass das Gespräch uneinholbar ist. Wir übernehmen ja die Ich-Personen des Originals, wir sagen nicht »er sagt« oder »sie sagt «, wir reden ja als deren »Ich«.

AS: Ich frage mich darüber hinaus, was für Entscheidungen in Bezug auf die Kameraeinstellungen gefällt wurden. Es ist doch interessant, dass Deleuze zusammen mit Félix Guattari sehr viel über das Gesicht und die Gesichthaftigkeit gearbeitet hat, über das Verhältnis von Sprache und Ge- 
sicht, über Sprechen und Gesicht. Und er sich dann einer Kamera aussetzt, die über eine Länge von mehr als sieben Stunden immer auf sein Gesicht und seinen Körper gerichtet ist, während er spricht. Also frage ich mich, was für Entscheidungen wohl dahinter standen, stundenlang Gilles Deleuzes Gesicht beim Sprechen zu filmen, ob das besondere Bewusstsein für das Verhältnis von Gesicht und Sprechen eine Rolle gespielt hat und ein bestimmter Effekt erzielt werden sollte. Ein Effekt, der uns jetzt wiederum entgeht, wenn wir nachträglich »gesichtslos« die deutsche Version sprechen.

HZ: Wir sitzen in der Falle der vorhandenen Aufnahme. Zwar setzen wir allein durch den Wechsel und die Überlagerung der Sprachen andere Akzente. Dennoch frage ich mich: Welchen Effekt macht es eigentlich, wenn für den Zuschauer der physiognomische und der stimmliche Ausdruck von einer surimpression überlagert wird, die durch die zweite Stimme aus dem Off erzeugt wird? Das heißt, da gibt es eine Art von Verzerrung. Wenn ich zum Beispiel die deutsche Stimme von John Wayne höre, drängt sich der Eindruck eines schweren Machos auf. Wayne aber hatte eine sanfte, fast weich fließende irische Stimme. Was ist da passiert?

Das heißt, ich defiguriere nicht nur das Sprechen von Deleuze, sondern auch sein Gesicht, seinen Ausdruck. Ähnliches gilt für Claire. Darüber muss man sich im Klaren sein. Wie nimmt man so etwas auf, wenn man das betrachtet? Was höre ich und wem höre ich wirklich zu?

AS: Wahrscheinlich haben wir durch unsere Stimmen den beiden auch ein anderes Gesicht gegeben. Wer spricht also zu wem? Wer sieht wen an? Ich habe mich außerdem gefragt, als was man das bezeichnen sollte, was die beiden in dieser Aufnahme machen. Kann man überhaupt von einem Dialog sprechen? Der Austausch zwischen den beiden hat ja eine Struktur vorgegeben bekommen durch das Alphabet, durch die Entscheidung, jeden Buchstaben des Alphabets zu benennen und dazu einen Begriff, eine Idee zu diskutieren. Das macht ihn doch zu einer sehr speziellen Form des Dialogs.

HZ: Ein monologue à deux?

AS: Geht es vielleicht um den Prozess des Sprechens selbst, der uns im Nachhinein erlaubt, ein Stück weit das Denken von Gilles Deleuze nachzu- 
vollziehen, genau in seiner Prozesshaftigkeit, in seinem Wandern? Denn es handelt sich hier ja kaum um ein Erklären von Begriffen, mit denen er in seiner philosophischen Vergangenheit gearbeitet hat, es ist keine Rückschau seiner Bücher, kein Interview über seine Karriere. Wie könnte man benennen, was es ist und worum es geht?

HZ: Ich finde es sehr wichtig und nützlich, sich darüber zu verständigen, womit man es da zu tun hat. Es geht doch um die Bestimmung des Genres oder der Form dessen, was uns da entgegen tritt. Die erste Bestimmung für mich ist nicht in der Dichotomie männlich/weiblich begründet, obwohl sie hier nicht unwesentlich ist, sondern vielmehr in der Atmosphäre der Vertrautheit zwischen den beiden. Das heißt, sie kennen und vertrauen einander, und es findet auch in einer Wohnung statt - offenbar in seiner Wohnung - es ist also etwas, das im privaten Raum, in der Geborgenheit stattfindet. Aber es weist weit über den privaten Raum hinaus. Zum anderen eröffnet es durch die Zeit, die sie sich lassen und die sie sich genommen haben, die Möglichkeit zu so etwas wie einer gemeinsamen Meditation. Also man sieht und spürt sie meditieren. Sie meditieren in dem Sinne, dass er lange und ausführlich nachdenkt, sich auf etwas einlässt, was ihm angeboten wird, womit er gelockt wird. Manchmal ist er auch etwas irritiert, obwohl er vielleicht selbst an der Wahl der Begriffe beteiligt war, aber jetzt soll er sich darauf wirklich einlassen, und er tut es dann auch, mit einer Ausnahme, glaube ich: »Wittgenstein«, wo nichts mehr geht. Der Überraschungseffekt ist also eigentlich relativ gering. Weil offensichtlich eine Vereinbarung stattgefunden hat. In diesem sehr freien Raum, den sie sich gegeben haben, findet kein Monolog statt. Deleuze ist ja nicht jemand, der vor sich hin monologisiert, sondern er spricht zu ihr. Ganz eindeutig. Er spricht nie in die Kamera. Die Kamera ist nah bei ihr, an ihrer Schulter, an ihrem Ohr gewissermaßen, also ich sehe das mal als eine Ohrposition, eine Mithörposition, oder auch in der Nähe ihres Auges: Die Tatsache, dass er zu ihr spricht und sie als eine Mithörende und eine Mitsprechende anerkennt und braucht, macht das Ganze bereits monolog-untauglich. Es ist tatsächlich ein Dialog der ungewöhnlichsten Form, da dieser hier nämlich in ihrem konzentrierten Zuhören besteht, das noch katalytisch verstärkt wird durch die Kamera. Also der Gestus des Zuhörenkönnens und -wollens ist außerordentlich stark und von ihm und ihr gleichermaßen gewollt - ohne das ginge es gar nicht. 
AS: Die beiden haben vor dem ABC, im Jahr 1977, ein gemeinsames Buch verfasst und produziert, das Dialoge betitelt ist. (Deleuze/Parnet 1977) Das Buch hat vier Kapitel, von denen jedes zwei Teile hat, und man begreift relativ schnell, ohne dass es genau gekennzeichnet wäre, dass Teil eins von Deleuze und Teil zwei von Parnet kommt. Gleich das erste Kapitel widmet sich der Frage »Was ist eine Unterhaltung und was nützt sie?«. Beide machen den Punkt stark, dass eine Unterhaltung im Grunde erreichen soll, den Schemata der, wie sie es nennen, binären Maschine, dem Machtapparat, etwas entgegenzusetzen. Die Binärmaschine arbeitet mit Schemata wie Frage/Antwort, männlich/weiblich, wissenschaftlich/nicht-wissenschaftlich. Bei Deleuze und Parnet sollen diese Oppositionen zugunsten eines »Werdens « abgelöst werden. »Werden« ist ja auch ein Begriff, der im ABC immer wieder auftaucht.

Wie kann dieser Begriff des Werdens für eine Unterhaltung verstanden werden? Und geht am Ende das auf, was die beiden hier unternehmen, die sich eben auch theoretisch sehr viel mit den Prozessen und Mechanismen des Gesprächs und der gemeinsamen Arbeit beschäftigt haben? Geht das im Falle des ABCs auf? Sie thematisieren immer wieder, was es heißt, zu zweit zu arbeiten und sich oberflächlichen und binären Strukturen zu entziehen, um anders denken, anders schreiben und anders sprechen zu können.

HZ: Das ganze Gespräch ist für mich das Bild eines weit verzweigten Werdens. Deleuze tastet sich an etwas heran, dessen Ende er im Grunde noch nicht kennt. Er stößt ins Offene vor in seiner Rede, in seinem Suchen, seinem Tasten, seinen verrückten Navigationen. Und sie fängt ihn manchmal auf. Da gibt es dieses leichte Kapern bei ihr. Ich habe manchmal den Eindruck, dass sie ihn sowohl kapert als auch wie eine Hebamme arbeitet. Sie entlockt ihm die Sprache.

AS: Dies scheint ihre Strategie zu sein, um dem System zu entkommen, das sie so kritisieren, das mit vorgefertigten Fragen und erwartbaren Antworten arbeitet.

Der Buchstabe F steht in der französischen Version des ABC für Fidelité, wird aber im Deutschen klugerweise mit »Freundschaft« übersetzt, und widmet sich genau dieser Multiplizierung der Autorschaft oder eben genau der Infragestellung eines singulären Autors oder Autorsubjekts. Claire Parnet geht vor allen Dingen auch auf die Freundschaft zwischen 
Gilles Deleuze und Félix Guattari ein und versucht anzuknüpfen an eine Tradition von Freundschaft und Freundespaaren, die auch gemeinsam irgendwie schöpferisch tätig sind.

HZ: Bei Fidelité, »Freundschaft«, taucht diese merkwürdige Erinnerung von ihm auf. Er zitiert die Zeile »Hast du einen Freund hinieden« aus dem Gedicht »Zwielicht « von Eichendorff. ${ }^{1}$ Hier heißt es, es gibt Stunden und es gibt Augenblicke, in denen selbst der beste Freund eine Gefahr darstellt. Das hat mich fast erschüttert, weil ich mir über die Intention von Deleuze, es in diesem Zusammenhang aufzurufen, immer noch nicht im Klaren bin. Freundschaft wird mit diesem Zitat düster.

AS: Er sagt ohnehin ein paar Dinge, die man beim Thema Freundschaft nicht erwartet. So zum Beispiel das offene Bekenntnis, von Guattari intellektuell gestohlen zu haben und die Hoffnung, dass Guattari das wiederum seinerseits bei Deleuze getan hat. Er hat ein Verständnis von Zusammenarbeit, das eben nicht darin aufgeht, dass man zum gleichen Thema schreibt und sich alles harmonisch zusammenfügt. Gedanken reihen sich vielmehr aneinander, Teile gehen in verschiedene Richtungen und treffen sich dann irgendwo wieder, Begriffe kommen auf und treten ins Verhältnis zu anderen - und man eignet sich dabei durchaus Gedanken des anderen an und wird einander zur Gefahr. Das war für mich eine überraschende Wendung dieses Freundschaftsthemas.

HZ: Wobei das Eigentümliche ist, dass wir Fidelité eigentlich nicht mit Freundschaft übersetzen würden, wenn man aber andererseits nur von Treue spricht, rutscht das gleich auf eine ganz andere Ebene. Es gibt offensichtlich etwas in der Intimität bestimmter Beziehungen, gerade von Leuten, die sich hauptsächlich mit dem Denken befassen, das auch gefahrvolle Momente beinhaltet.

1 | Die dritte Strophe aus "Zwielicht» von Joseph von Eichendorff (1815) lautet: Hast du einen Freund hinieden, Trau inm nicht zu dieser Stunde, Freundlich wohl mit Aug und Munde, Sinnt er Krieg im tückschen Frieden. 
AS: Für mich stellt sich im konkreten Fall des ABC die Frage, worin genau Claire Parnets Anteil besteht. Es ist offensichtlich, dass sie es konzipiert hat, dass sie jedem Buchstaben Begriffe zugeordnet hat, auch in Absprache mit ihm. Man fragt sich dabei immer wieder, ob sie auf etwas Bestimmtes hinaus möchte, ob sie etwas Bestimmtes von ihm hören will. Gerade an Stellen, an denen sie etwas biographischer fragt oder ihn dahin locken will, über sich selbst, über seine Kindheit, seine gesundheitliche Verfassung oder auch seinen Bezug zu 1968 zu erzählen, an diesen Stellen scheint es manchmal, als hätte sie eine Art größeren Plan. Als wollte sie ihn dazu bringen, Aussagen über sich in diesem »Testament« festzuhalten. Aber sie tut dies, indem sie immer wieder neue Linien für das Gespräch zieht.

HZ: Schon der zweite Buchstabe B - Boisson, im Deutschen »Trinken«, das heißt im Klartext: Alkoholsucht ist ein sehr biographisches Thema. Und er spricht nicht nur über seine - überwundene - Trinksucht mit fast analytischer Klarheit: »Heute höre ich auf, um morgen wieder anfangen zu können«, sondern er holt plötzlich weit aus und kommt auf die von ihm bewunderten amerikanischen Schriftsteller Scott Fitzgerald und Thomas Wolfe zu sprechen.

AS: Und hier nimmt Claire ganz entschieden den Faden auf, wenn sie über die Verbindung von literarischer Arbeit und Trinken sagt: »daß es dem Alkohol eine Lebensmacht gab, die zu stark für sie war, zu mächtig, wenngleich nur sie diese Macht wahrnehmen konnten.« Und Deleuze spielt dann auf einen für ihn sehr eigentümlichen Unterscheid zwischen den amerikanischen und den französischen Schriftstellern an.

HZ: Ja, er sagt, sie, die Franzosen hätten nicht denselben Blick auf das Schreiben wie die Amerikaner; denen ginge es nämlich darum »etwas zu sehen, etwas zu sehen, was die andern nicht sehen.«

Erstaunlich und sehr bewegend ist auch, wie er das Vorkriegs-Panorama des Abstiegs seiner Familie aus dem wohlhabenden 17. (Pariser) Arrondissement entwirft. Als würde er die Ballistik eines immer tiefer sinkenden Flugkörpers beschreiben...

AS: In diesem Zusammenhang, es ist der Buchstabe E - Enfance, »Kindheit«, erinnert Deleuze an etwas, das man sich heute kaum mehr vorstellen kann: die Durchsetzung des ersten bezahlten Urlaubs für Arbeiter 
und kleine Angestellte in Frankreich. Deleuze beschreibt, wie diese Menschen am Strand von Deauville auftauchen, dem Urlaubsort der Pariser Bourgeoisie, und wie das Großbürgertum vor den eigenen Landsleuten erschrickt und entsetzt ist.

Claire Parnet stellt mit der Stoßrichtung ihrer Fragen immer wieder Bezüge zum Außerphilosophischen oder Nicht-Philosophischen her, die für seine Arbeiten ganz eminent wichtig sind. Zwischen einer philosophischen Arbeit und einem Leben außerhalb davon wird nicht getrennt, sondern Parnet stellt ein Verhältnis zwischen beiden her, indem sie eine Auswahl von Begriffen wie »Kindheit«, »Krankheit«, »Tennis« oder »Oper« trifft und das Außen immer wieder mit herein bringt, genau wie er. Ihr scheint zum Beispiel der Bezug zu den politischen Bewegungen und Ereignissen seiner Zeit besonders wichtig und sie verleitet ihn, deren Bedeutung für seine philosophische Arbeit zu formulieren.

HZ: Ja, sie verführt ihn schon, das ist schon eine Art von sanfter Verführung und Lockung. Sie ermutigt ihn, über Dinge zu sprechen, die nicht primär philosophischer Art sind, und er geht darauf ein, wenn er diese schöne Ausführung macht: Man muss nicht Philosoph sein, um philosophisch zu denken. Es ist eine wunderbare Formel, die er da entwickelt.

Oder wenn sie ihn dazu bringt, in knapper Form den Anti-Ödipus ${ }^{2}$ darzustellen. Ich finde das eine unglaubliche Stelle. Er sagt ungefähr: Das war etwas ganz Einfaches, was Félix und ich da gemacht haben. Und dann erklärt Deleuze, wie Guattari und er die gemeine Vorstellung von Wunsch zurechtrücken. Man begehrt nicht irgendetwas oder irgendjemanden, sondern immer ein Ensemble. Er sagt, die zentrale Frage im Anti-Ödipus lautet: »Wie müssen die Beziehungen zwischen Elementen beschaffen sein, damit ... Wunsch entstehen kann, damit (diese Elemente) begehrenswert werden?« Das ist zum Beispiel eine Sache, von der ich denke, dass er sie alleine ohne Anstoß nicht gesagt hätte.

AS: Ich glaube, dass das ein ganz wesentlicher Punkt ist. Das Zusammenspiel von Deleuze und Parnet besteht darin, Philosophie als eine Praxis $\mathrm{zu}$ verstehen, die weit darüber hinausgeht, philosophiegeschichtliche Bücher zu schreiben oder sich an einzelnen Philosophen abzuarbeiten. Ihre Philosophie dagegen steht in unmittelbarem Bezug zum Außerphiloso-

2 | Deleuze/Guattari 1972. 
phischen und bedeutet nicht nur die Fähigkeit, neue Begriffe zu schaffen, sondern diese auch auf konkrete Phänomene zu beziehen. Hier sehe ich einen der Kernpunkte des ABC-Projektes, auf den beide großen Wert legen. Drei Jahre, bevor sich Deleuze und Guattari gemeinsam fragen, was Philosophie ist ${ }^{3}$, löst das ABC die strikte Trennung von Philosophie und Nicht-Philosophie auf. Es ist auffällig, dass keiner der Buchstaben sich direkt auf ein philosophisches Konzept bezieht, es gibt höchstens ein paar wenige Buchstaben, die nach einzelnen Philosophen benannt sind, wie K für Kant und W für Wittgenstein, über den er ja nicht reden will. Aber es gibt wiederum kein B für Bergson, kein $\mathrm{N}$ für Nietzsche und kein $\mathrm{S}$ für Spinoza. Philosophische Konzepte ergeben sich aus der philosophischen Praxis und dem Sprechen heraus, Philosophie ist eine Praxis und ein Produzieren von Fragen und Begriffen. Sie reicht weit über den akademischen Rahmen hinaus und berührt Essensgewohnheiten, Fragen des Stils, Fernsehdebatten oder das Reisen. Ich finde, diese Praxis führen die beiden in ihrem Gespräch sehr gut vor.

Gleichzeitig liegt für uns eine Möglichkeit darin, dass es Deleuze und Parnet offensichtlich nicht darum gegangen ist, einen bestimmten Punkt zu machen, den wir jetzt übersetzen müssen. Vielleicht liegt genau darin eine besondere Ethik, sich gegen einen festen Ausgangspunkt und ein festgelegtes Ziel zu sperren, das dann von A bis Z konsequent verfolgt wird. Auch wenn ein ABC vielleicht zunächst nahe legt, dass es vom ersten bis zum letzten Buchstaben eine Entwicklung gibt, die nachvollzogen werden muss. Aber genau dagegen verwehren Deleuze und Parnet sich. Hierin liegt die Herausforderung und Schwierigkeit für uns, aber auch die Möglichkeit, heute wieder ansetzen zu können. Und damit dieses einzigartige Dokument immer wieder zu öffnen und zugänglich zu machen.

ABÉCÉDAIRE - Gilles Deleuze von A bis Z. Ein Film von Pierre-André Boutang. Konzeption und Interview von Claire Parnet, übers. v. Valeska Bertoncini, hg. v. Valeska Bertoncini und Martin Weinman, ko-produziert von Zweitausendeins und Absolut Medien.

D 2009 (F 1988-89/1996).

3 | Deleuze/Guattari 1991. 


\section{LITERATUR}

Deleuze, Gilles/Parnet, Claire: Dialoge, übers. v. B. Schwibs. Frankfurt a. M. [1977] 1980.

Deleuze, Gilles/Guattari, Félix: Anti-Ödipus. Kapitalismus und Schizophrenie 1, übers. v. B. Schwibs, Frankfurt a. M. [1972] 2008.

Deleuze, Gilles/Guattari, Félix: Was ist Philosophie?, übers. v. B. Schwibs/J. Vogl, Frankfurt a. M. [1991] 2007.

Eichendorff, Joseph von: »Zwielicht«, in: ders., Sämtliche Werke des Freiherrn Joseph von Eichendorff, Gedichte, Bd. 1,1, hg. v. Harry Fröhlich/ Ursula Regener. Stuttgart/Berlin/Köln [1815] 1993, S. 11-12. 